\title{
Assessing Psychological Symptoms in Recent Immigrant Adolescents
}

\author{
Sita G. Patel • Melissa A. Kull
}

Published online: 7 September 2010

(c) The Author(s) 2010. This article is published with open access at Springerlink.com

\begin{abstract}
Immigrant youth are often exposed to numerous psychosocial stressors, placing them at risk for psychological distress. Little research assesses psychopathology in this population during early stages of acculturation. This study compared student and teacher reports of psychological symptoms in a diverse sample of recently immigrated youth. Students $(N=174)$ attended public high schools in a northeastern city. Students and teachers independently completed the Achenbach System of Empirically Based Assessment, and four DSM-derived subscales were explored. Psychological symptoms among immigrant students were higher than normative rates. Across all subscales, teacher ratings of student symptoms were significantly lower than student self-reported symptoms, and this difference was larger than that found in a normative sample. Results suggest that many immigrant youth experience psychological problems but may not be perceived as being in distress. Therefore, the most effective assessment approach may be active screening, rather than relying on self initiated help-seeking or teacher observation alone.
\end{abstract}

Keywords Acculturation - Assessment · Adolescents . Psychopathology

S. G. Patel $(\bowtie)$

Department of Psychiatry, University of California, 2727

Mariposa Street, Suite 100, San Francisco, CA 94110, USA

e-mail: sita.patel@ucsf.edu

M. A. Kull

Department of Applied Psychology, New York University,

New York, NY, USA

\section{Background}

Currently, there are 37.2 million foreign born residents in the United States, approximately 2.7 million $(7.3 \%)$ of whom are between ages of 5 and 17 [1]. Many immigrant youth living in low income, urban settings are exposed to distinct stressors related to their experience during immigration, the adjustment and acculturation process, and exposure to environmental risks associated with poverty $[2,3]$. However, it remains unclear whether recentlyimmigrated youth fare worse than their mainstream, non-immigrant counterparts. Considerable evidence demonstrates a correlation between exposure to acculturative stressors, such as immigration-related trauma or ethnic discrimination, and the presence of psychological problems like anxiety and depression, in adult [4] and youth immigrants [5].

While these studies start to identify the prevalence of mental health problems among immigrant youth, studies on the psychological health of this population remain inconclusive, with some showing immigrant youth doing better than their mainstream peers, and others finding increased rates of symptoms such as anxiety, depression, aggression, low self-esteem, dependency, and poor peer relations $[6,7]$. One possible problem with previous studies of immigrant health may be sampling limitations. Many studies look exclusively at one ethnic group, or include immigrants with a wide range of time living in the US, or also include US-born children of immigrants in their samples. In narrowing this broad scope, we may find that recently immigrated youth are at particularly high risk, because they are in the early stages of adapting to a new environment and coping with the losses of leaving a host country behind. Conversely, it may be that recently immigrated youth retain protective elements of traditional 
culture that help them cope with stressors. However, no studies to date use a narrow post-immigration time frame to limit their sample.

Most studies also rely exclusively on self-report, so it remains unclear if psychological problems are perceived by others. School professionals provide an important source of information to help identify and report psychological and behavioral problems among school-aged youth, and mental health providers agree that teachers are one of the most reliable sources for reporting students' symptoms [8]. Recently immigrated youth may face communication and cultural barriers that impede accurate assessment by their teachers, who are expected to interact with students from a wide range of cultural backgrounds. However, little research has explored teachers' assessment accuracy of psychopathology among recently immigrated youth. To address these gaps in existing evidence, this study compared rates of psychological symptoms and teacher assessment accuracy between recently-immigrated youth and a normative sample.

\section{Methods}

\section{Participants}

Participants $(N=174)$ were recruited from two international public high schools created specifically for recent immigrants (students within three years of immigration may enroll). The student bodies of these schools represent 61 different countries of origin, and all instruction is in English. Fifty-three percent of the sample identified as female. Sixteen percent of participants were enrolled in 9th grade, 25\% 10th grade, 34\% 11th grade, and 25\% 12th grade. In keeping with the school population, study participants represented multiple countries of origin, with $28 \%$ from the Caribbean, 28\% from Latin America, 21\% from East Asia, 11\% from South Asia, 6\% from Europe, and 6\% from the Middle East, with an average time since immigration of 4 years, and a range of $0-8$ years.

\section{Data Collection}

Following approval from the state Department of Education and the human subjects research review board of a major university, the study was presented to students in their classrooms, with parental consent forms (translated into parents' primary languages) distributed to interested students. Students who returned parental consent forms and assented to participate came to the schools' computer labs during their free periods to complete an online survey, in English, with research staff assisting as needed. Teachers of participating students independently completed teacher report forms online.
Measures

\section{Demographics}

Various items were adapted from the Immigrant Adolescent Questionnaire [4] to ascertain characteristics of the sample, such as gender, grade, years in the US, country of origin, religion, and citizenship status.

\section{Psychological Adjustment}

The Achenbach System of Empirically Based Assessment (ASEBA) [9] was used to evaluate participants' psychological and behavioral adjustment. This comprehensive measure assesses depression, anxiety, psychosomatic symptoms, conduct problems, and school and social adjustment, and it has been validated in over 20 different countries [10]. The ASEBA allows for multiple informants by including both a Youth Self-Report (YSR) and a Teacher-Report Form (TRF). For this study, four subscales, or "syndrome profiles" were calculated, indicating problems that form a particular syndrome: affective problems (13 items; YSR $\alpha=.8$; TRF $\alpha=.9$ ), somatic problems (7 items; YSR $\alpha=.8$; TRF $\alpha=.9$ ), anxiety problems (6 items; YSR $\alpha=.6$; TRF $\alpha=.9$ ), and attention deficit hyperactivity disorder (ADHD) problems ( 7 items; YSR $\alpha=.7$; TRF $\alpha=.9$ ).

The standardized ASEBA measure designates three classifications of cumulative $T$-scores: $<63=$ normal; $63-69=$ borderline; and $>70=$ clinically significant. Scores in the borderline or clinically significant classifications are considered to be of clinical concern and indicate the need for further assessment and/or treatment. Participating students were compared to a representative normative sample from the ASEBA literature [9].

\section{Analysis and Results}

To examine the number of participating students who fell above the cutoff for clinical concern (borderline/clinically significant $T$-scores), cumulative scores were calculated for each of the four subscales, and one-sample $t$-tests were run comparing sample scores with normative populations derived from the ASEBA's standardization procedures. The percentage of participating students with scores above the cutoff was $30 \%$ for Affective problems $(n=52), 22 \%$ for Somatic problems $(n=38), 12 \%$ for anxiety problems $(n=21)$, and $7 \%$ for ADHD problems $(n=12)$. As shown in Table $1, t$ tests found that sample means were significantly higher than normative means in all domains except ADHD, such that that participating students reported significantly more symptoms of internalizing psychopathology across three DSM domains. 
Table 1 Recent immigrant youth report more internalizing symptoms than normative population

\begin{tabular}{lllll}
\hline & $\begin{array}{l}\text { Sample mean } \\
\text { Mean (SD) }\end{array}$ & $\begin{array}{l}\text { Population mean } \\
\text { Mean (SD) }\end{array}$ & $t$ & $d f$ \\
\hline Affective problems & $59.32(8.01)$ & $54.30(5.06)$ & $8.27^{*}$ & 135 \\
Anxiety problems & $56.40(6.45)$ & $54.30(5.23)$ & $4.7^{*}$ & 135 \\
Somatic problems & $58.35(8.62)$ & $54.20(4.86)$ & $6.35^{*}$ & 135 \\
ADHD problems & $54.55(5.65)$ & $54.41(5.42)$ & -47 & 135 \\
\hline
\end{tabular}

$* P<.0001$

Table 2 Recent immigrant youth report more psychological problems than teachers perceive

\begin{tabular}{lclll}
\hline & $\begin{array}{l}\text { Student mean } \\
\text { Mean (SD) }\end{array}$ & $\begin{array}{l}\text { Teacher mean } \\
\text { Mean (SD) }\end{array}$ & $t$ & $d f$ \\
\hline Affective problems & $59.32(8.01)$ & $52.67(5.06)$ & $9.8^{*}$ & 135 \\
Anxiety problems & $56.4(6.45)$ & $52.99(5.23)$ & $5.5^{*}$ & 135 \\
Somatic problems & $58.35(8.62)$ & $51.66(4.86)$ & $8.31^{*}$ & 135 \\
ADHD problems & $54.55(5.42)$ & $51.67(2.89)$ & $5.89^{*}$ & 135
\end{tabular}

$* P<.0001$

Paired sample $t$ tests were also used to assess teacher assessment accuracy by comparing youth self-reported symptoms with teacher reports of student symptoms. As shown in Table 2, teacher ratings of students' symptoms were significantly lower than student self-reported symptoms across all four subscales. While small gaps do exist between student and teacher reports within the normative comparison sample, reporting differences for participating teachers and students in the study sample were notably larger.

\section{Discussion}

Results are consistent with previous research showing that immigrant youth experience elevated levels of psychological symptoms. This finding is confirmed in a unique sample of recently immigrated youth from a representative range of cultural backgrounds, and across three of four DSM-derived subscales, all representing internalizing problems. This suggests that the early stages of immigration is a particularly sensitive time, when individuals are actively adapting to a new environment and recovering from losses of leaving their country of origin. Recently immigrated youth may be at particular risk for undetected internalizing problems. Studies exploring explanations for psychological problems during acculturation suggest that adolescents' perceptions of discrimination, insufficient language proficiency, and decreased parental support may all contribute to difficulties following immigration [5].
Interestingly, ADHD problems were not higher in this sample of immigrant adolescents, although behavioral problems have previously been found to be higher among younger immigrant children [6]. This result could be agerelated, suggesting that immigrant teens tend to internalize more than externalize their psychological conflict. The result could also be context-dependent: this sample attends a small school in which behavioral problems may be less tolerated and more quickly addressed than in larger school settings with greater student to teacher ratios.

Across all four subscales, teachers reported significantly lower levels of youth symptoms than youth self-reported, even for externalizing symptoms, which should be more readily observable. Further, this gap is greater than what is found in a normative sample. This set of results suggests that recent immigrant youth experience a range of psychological problems, but may not be observed as being in distress by those around them. It may be that language or cultural differences between immigrant students and their teachers prevent effective communication of sensitive psychological issues. It may also be that immigrant youth seek help from adults at a lower rate than their mainstream peers, or that teachers may misinterpret or not fully understand culture-specific ways of expressing emotional distress.

Several limitations to the present study merit further exploration. First, the measures were administered in English, a procedure that excluded students who were the least acculturated. Second, the sample size was too small to compare findings across ethnic groups. Future studies based on this line of research should include a large enough sample size to permit analyses within and across cultural groups, as well as including recent immigrants without English proficiency by translating measures into languages of origin.

The present study suggests that as a whole, recently immigrated youth are at elevated risk for internalizing psychological problems, and that these problems are not readily observed by their teachers. Such results suggest that services for this vulnerable group of students should include school-based screening and assessment procedures in order to effectively identify and treat mental health problems within this under-served population.

Acknowledgments This research was supported in part by U.C. Berkeley's Cultural Psychology Training Grant, and NIMH grants MH067562 and MH074500. The authors wish to thank Rhona Weinstein, Jennifer Alvidrez, Martha Shumway, Dawn Kaiser and Megan Moore.

Open Access This article is distributed under the terms of the Creative Commons Attribution Noncommercial License which permits any noncommercial use, distribution, and reproduction in any medium, provided the original author(s) and source are credited. 


\section{References}

1. US Census Bureau. S0501 selected characteristics of the native and foreign-born populations: American Community Survey. Washington, DC: Government Printing Office; 2005-2007.

2. Berry JW. Psychology of acculturation. In: Goldberger NR, Veroff JB, editors. The culture and psychology reader. New York: New York University Press; 1998. p. 457-88.

3. Gonzales NA, et al. Validation of a multicultural events scale for urban adolescents. Paper presented at the biennial conference of the society for community action and research. Chicago, IL; 1995.

4. Berry JW, et al. Comparative studies of acculturative stress. Int Migr Rev. 1987;21:491-511.

5. Leibkind K, Jasinskaja-Lahti I. Acculturation and psychological well-being among immigrant adolescents in Finland. J Adolesc Res. 2000;15:446-69.
6. Aronowitz M. The social and emotional adjustment of immigrant children: a review of the literature. Int Migr Rev. 1984;18: 237-57.

7. Stodikska M. Adaptation processes among young immigrants: an integrative review. J Immigr Refug Stud. 2008;6(1):34-59.

8. Loeber R, et al. Mental health professionals' perception of the utility of children, mothers and teachers as informants on childhood psychopathology. J Clin Child Psychol. 1990;19:136-43.

9. Achenbach TM, Rescorla LA. Manual for the ASEBA school-age forms and profiles: an integrated system of multi-informant assessment. Burlington, VT: University of Vermont; 2001.

10. Achenbach TM, Becker A, Döpfner, M, Heiervang E, Roessner V, Steinhausen HC, Rothenberger A. Multicultural assessment of child and adolescent psychopathology with ASEBA and SDQ instruments: research findings, applications, and future directions. J Child Psychol Psychiatry. 2008;49(3):251-75. 